\title{
MODELING THE DYNAMICS OF A SYSTEM OF
}

METROPOLITAN AREAS: A DEMOECONOMIC APPROACH

P. Gordon

J. Ledent

RR-80-8

March 1980

Reprinted from Environment and Planning A, volume 12 (1980)

INTERNATIONAL INSTITUTE FOR APPLIED SYSTEMS ANALYSIS Laxenburg, Austria 
Research Reports, which record research conducted at IIASA, are independently reviewed before publication. However, the views and opinions they express are not necessarily those of the Institute or the National Member Organizations that support it.

Reprinted with permission from Environment and Planning $A$ 12:125-133. Copyright @ 1980 Pion Limited.

All rights reserved. No part of this publication may be reproduced or transmitted in any form or by any means, electronic or mechanical, including photocopy, recording, or any information storage or retrieval system, without permission in writing from the copyright holder. 


\section{FOREWORD}

Declining rates of national population growth, continuing differential rates of regional economic activity, and shifts in the migration patterns of people and jobs are characteristic empirical aspects of many developed countries. In several instances, they have combined to bring about a relative (and in some cases absolute) population decline in highly urbanized areas, e.g., New York, Tokyo, and Stockholm. In other cases, e.g., Houston, Miami, and Moscow, they have brought about rapid metropolitan growth.

The objective of the Urban Change Task in IIASA's Human Settlements and Services Area is to bring together and synthesize available empirical and theoretical information on the principal determinants and consequences of such urban growth and decline.

This paper argues for a demoeconomic approach to the evolution of a system of metropolitan areas. It proposes a complete dynamic model centered on the roles of trade and migration while considering interregional as well as interregional effects.

A list of related publications in the Urban Change Series appears at the end of this paper.

ANDREI Rogers

Chairman

Human Settlements and Services Area 


\title{
Modeling the dynamics of a system of metropolitan areas: a demoeconomic approach
}

\author{
P Gordon I, J Ledent \\ International Institute for Applied Systems Analysis, Laxenburg, Austria \\ Received 28 November 1978, in revised form 7 August 1979
}

\begin{abstract}
This paper proposes a complete dynamic model of a system of metropolitan areas interacting through economic and demographic links, namely trade and migration respectively. It not only considers interregional effects (through an interregional input-output submodel) but also intraregional effects (through a set of Garin-Lowry submodels). In addition it allows the simultaneous determination of migration rates, labor-force-participation rates, and unemployment rates. Suggestive of the demoeconomic approach, this model also reveals the methodological difficulties that such an approach implies.
\end{abstract}

The reasons for a demoeconomic approach to urban and regional modeling are based on the simple fact that economic and demographic variables interact. In spite of this, there exist virtually no interregional economic models having a demographic dimension. An exception to this is the Urban Institute's model of employment growth and migration in US metropolitan areas (Alperovich et al, 1975; 1977).

In this paper we propose an alternative model in which the economic side is based on the standard interregional input-output structure advocated by Isard (1960) rather than on an econometric specification as in the Urban Institute model. In addition our model features several important additional characteristics:

(1) it considers intraregional effects of economic development;

(2) it treats economic and demographic variables in accordance with Miron's (1978) suggestion that migration rates, unemployment rates, and labor-force-participation rates must be endogenously and simultaneously determined; and

(3) it allows for the interaction of the life cycle of the population with the life cycle of cities and that of a system of cities. The fact that these cycles interact may be expected: an aging population, for example, affects urban services and the land-use pattern of services. At the same time, the age structure decides migratory flows, which in turn affect the age structure in the various cities.

The presentation of our model is carried out in three stages. In section 1, which is concerned with a unique metropolitan area, we critically review the traditional GarinLowry model (Lowry, 1964; Garin, 1966) by presenting its most restrictive attributes and we introduce a dynamic version eliminating the limitation of an infinitely elastic labor supply (through the explicit consideration of migration). But in section 2, with the realization that a metropolitan area is open to trade, we enlarge the scope of our analysis to a system of local and interregional relationships: this is carried out by linking a set of Garin-Lowry models, one for each metropolitan area, with the help of an interregional input-output model. Then in section 3 we present our linking of the economic and demographic sectors in accordance with the principles set forth in Ledent (1978) and Ledent and Gordon (1978). We also introduce an age breakdown of population by use of the multiregional cohort-survival model of population growth (Rogers, 1975) as an underlying framework.

II Present address: Department of Economics, University of Southern California, Los Angeles, California 90007, USA. 
All of this leads to a complete dynamic and demoeconomic model of a system of metropolitan areas fusing three analytical tools (the Garin-Lowry model, the interregional input-output model, and the multiregional cohort-survival model). These are too often used independently. Yet their linearity makes their desirable combination easily possible.

1 The Garin-Lowry model: review and extension

In brief, the Garin-Lowry model is a model of spatial activity allocation in a metropolitan area based on the urban economic-base concept. Suppose that this area is partitioned into $r$ zones and let $e_{\mathrm{b}}(t), e_{\mathrm{nb}}(t)$, and $e(t)$ be three $r \times 1$ vectors describing the spatial allocation of basic, nonbasic, and total employment respectively at time $t$. Then

$$
e(t)=e_{\mathrm{b}}(t)+e_{\mathrm{nb}}(t) \text {. }
$$

The pattern of zonal residential locations is linked to the zonal set of employment locations through

$$
w(t)=\mathrm{FQ} e(t),
$$

where

$w(t)$ is an $r \times 1$ vector allocating population to zones,

F is an $r \times r$ diagonal matrix of zonal population-employment ratios, and

Q is an $r \times r$ work-to-home trip probability-distribution matrix.

The pattern of zonal locations of nonbasic activities is linked to the zonal residential locations through

$$
e_{\mathrm{nb}}(t)=\operatorname{PB} w(t),
$$

where

$\mathbf{P}$ is an $r \times r$ home-to-service trip probability-distribution matrix, and

B is an $r \times r$ diagonal matrix of zonal nonbasic employee-per-person ratios.

Combining equations (1) through (3) eventually leads to

$$
e(t)=(\mathrm{I}-\mathrm{PBFQ})^{-1} e_{\mathrm{b}}(t),
$$

where $\mathbf{I}$ is the $r \times r$ identity matrix, and

$$
w(t)=\mathrm{FQ}(\mathrm{I}-\mathrm{PBFQ})^{-1} e_{\mathrm{b}}(t),
$$

where $e_{\mathrm{b}}(t)$ is stipulated exogenously.

As such, the Garin-Lowry model has the following attributes.

1. It is based on the overly simple dichotomy suggested by the economic-base concept.

2. It is static.

3. It assumes an infinitely elastic supply of laborers (for example, through migration).

4. It presumes that all multiplier effects are local and not interregional.

5. It posits that regions export without importing.

6. It aggregates over the implicit population-cohort groups.

All of these limitations should be eliminated for our objectives to be realized. First we note that limitations 1 and 6 can be dealt with by incorporating the suggestions of Macgill (1977) and Schinnar (1978): the first has formulated the basic framework of the Garin-Lowry model as an input-output model and extended it to include full sectorial relationships; the second has introduced an age breakdown into the model. Thus we concentrate here on attributes 2 and 3 (in this section) and 4 and 5 (in the next section), although in the end we will propose a complete model removing all the limitations listed here. At this point we simply introduce migration in order to eliminate the assumption of an infinitely elastic supply of laborers. 
Let $p(t)=i^{\mathrm{T}} w(t)$, where $i$ is the $r \times 1$ vector all of whose components are one and $\mathrm{T}$ is the transpose operator, be the total population of the metropolitan area. Then

$$
p(t)=[1+n(t)] p(t-1)+M(t),
$$

where $n(t)$ is the (exogenous) rate of natural increase and $M(t)$ is the total net in-migration flow. The latter may be determined by the following equation, in which the net in-migration rate is shown to depend on an endogenous variable (the relative increase in total employment lagged one year) as well as on exogenous variables (for example, the national unemployment rate):

$$
m(t)=\frac{M(t)}{p(t-1)}=m\left[\frac{E(t-1)-E(t-2)}{E(t-2)}, \bar{u}(t-1)\right],
$$

where

$E(t-2)=i^{\mathrm{T}} e(t-2)$ is the total regional employment at time $t-2$, and $\bar{u}(t-1) \quad$ is the national unemployment rate at time $t-1$.

Thus exogenous variables and lagged values of endogenous variables determine net in-migration through equation (7) and then population through equation (6). It is precisely this treatment of the linkage between economic and demographic variables which introduces the dynamic character whose desirability was specified earlier.

Equation (5) of the Garin-Lowry model is still valid, but F, the matrix of population-employment ratios, is now endogenous and thus a time subscript is attached to it:

$$
\boldsymbol{w}(t)=\mathrm{F}_{t} \mathrm{Q}\left(\mathrm{I}-\mathrm{PBF}_{t} \mathbf{Q}\right)^{-1} e_{\mathrm{b}}(t) .
$$

Then the total population of the metropolitan area can also be obtained by premultiplying both sides of equation (8) by $i^{\mathrm{T}}$ :

$$
p(t)=i^{\mathrm{T}} \mathbf{F}_{t} \mathbf{Q}\left(\mathbf{I}-\mathbf{P B F}_{t} \mathbf{Q}\right)^{-1} e_{\mathrm{b}}(t) .
$$

If zonal population-employment ratios keep the same structure over time, we have

$$
\mathrm{F}_{t}=\alpha_{t} \mathrm{~F}_{t-1},
$$

where $\alpha_{t}$ is a coefficient identical for all zones. Substituting equation (10) into equation (9) and equating the result with the known value of $p(t)$, we get the following scalar equation:

$$
\alpha_{t}=\frac{p(t)}{i^{\mathrm{T}} \mathrm{F}_{t-1} \mathbf{Q}\left(\mathrm{I}-\alpha_{t} \mathrm{PBF}_{t-1} \mathrm{Q}\right)^{-1} e_{\mathrm{b}}(t)},
$$

which yields $\alpha_{t}$ implicitly and thus the solution of the model.

To obtain $\alpha_{t}$ an iterative method can be used as follows. Calculate a first estimate, $\alpha_{t}^{(1)}$, of $\alpha_{t}$ by replacing $\alpha_{t}$ by one in the right-hand side of equation (11). Then obtain an improved estimate by replacing $\alpha_{t}$ by $\alpha_{t}^{(1)}$ in the right-hand side of equation (11), etc. This procedure is continued until convergence is achieved.

Up to this point we have shown that the coupling of a traditional Garin-Lowry model with a simple migration model such as that in equation (7) removes limitations 2 and 3.

\section{Extension of the Garin-Lowry model to a system of metropolitan areas}

We now extend the scope of the analysis to a country of $R$ regions in which each region $i$ consists of $r_{i}$ economic subregions $\left(r_{i}=1\right.$ in the case of nonmetropolitan regions). The total number of economic subregions, $\sum_{i=1}^{R} r_{i}$, is denoted by $T$. The economic-base concept is abandoned: we deal with $S$ economic sectors and introduce an interregional input-output model. 
The first equation shows the derivation of final demands as the sum of consumption and government spending, which is taken as exogenous:

$$
f(t)=\mathbf{H} p(t-1)+g(t),
$$

where

$f(t) \quad$ is an $R S \times 1$ vector of final demand,

$\mathbf{H} \quad$ is an $R S \times R$ matrix of consumption coefficients.

$\boldsymbol{p}(t-1)$ is an $R \times 1$ vector of regional total populations, and

$g(t) \quad$ is an $R S \times 1$ vector of regional government spending-in the case of an open country, $g(t)$ includes net foreign exports.

The second equation supposes that, within each region and for each sector, employment corresponding to final demand is assigned to subregions according to

$$
e^{(0)}(t)=\mathrm{D} f(t),
$$

where

$e^{(0)}(t)$ is a $T S \times 1$ vector of employment corresponding to final demand, and

D is a TS $\times$ RS matrix of employment allocations.

Imports and exports are not a part of final demand in an interregional input-output structure. For reasons that will become apparent, we do not form a Leontief inverse but, rather, solve the problem of production by summing the various rounds of production. The first round of production due to interregional (final) demand is specified as

$$
x^{(1)}(t)=\mathrm{A} f(t)=\mathrm{AC}_{1} e^{(0)}(t),
$$

where

$x^{(1)}(t)$ is an $R S \times 1$ vector of the first round of production requirements due to interregional final demands,

A is an $R S \times R S$ matrix of technical coefficients (1), and

$\mathrm{C}_{1} \quad$ is an $R S \times T S$ matrix consolidating sectoral employments over the subregions.

Note that $\mathrm{C}_{1} \mathrm{D}=\mathrm{I}$.

It is also important to recall that the matrix of technical coefficients would be partitioned into intraregional production requirements on the main diagonal block and trade requirements in the off-diagonal blocks. Yet here we choose to delete the diagonal blocks because intraregional multiplier effects are taken care of by the Garin-Lowry model extended by Macgill (1977). To add the latter to the standard Isard (1960) input-output model would introduce double counting.

Within each region and for each sector, incremental employment is assigned to subregions in roughly the proportions of such employment already there; that is,

$$
E^{(1)}(t)=\mathrm{D} x^{(1)}(t) \text {, }
$$

where $E^{(1)}(t)$ is an $R S \times 1$ vector of the first round of subregional employments.

The next equation of the model is a generalization of the equation used by Garin (1966) to show the complete internal multiplier effects of the (spatially allocated) change in final demand. The left-hand side, then, shows the complete change in sectoral employments for all subregions of all regions due to the first-round employment effects only. We are looking at all the local iterations which have taken place as a result of the first iteration of the interregional model. We have that

$$
e^{(1)}(t)=\mathbf{K}_{t} E^{(1)}(t),
$$

(1) One of the difficulties in demoeconomic modeling is that many demographic parameters are more stable than the economic parameters. In the present instance, then, a ten-year simulation might have to be accomplished with the aid of a second but updated input-output coefficient matrix, where the second matrix is inserted into the model after, say, half the period of simulation. 
where

$e^{(1)}(t)$ is a TS $\times 1$ vector of additional local employment due to the first iteration of the interregional model, and

$\mathbf{K}_{t} \quad$ is a $T S \times T S$ block-diagonal matrix whose typical diagonal block (relating to each region) is a matrix generalizing the inverse matrix appearing in equation (4) of the Garin-Lowry model, namely

$$
\left(\mathrm{I}-\mathrm{P}^{i} \mathrm{~B}^{i} \mathbf{F}_{t}^{i} \mathbf{Q}^{i}\right)^{-1} \text {, }
$$

where

$\mathrm{P}^{i}$ is an $S r_{i} \times S r_{i}$ home-to-shopping trip probability-distribution matrix,

$\mathrm{B}^{i}$ is an $S r_{i} \times r_{i}$ diagonal matrix of zonal nonbasic employee-per-person ratios defined for each sector,

$\mathrm{F}_{t}^{i}$ is an $r_{i} \times r_{i}$ diagonal matrix of zonal population-employment ratios, and

$\mathrm{Q}^{i}$ is an $r_{i} \times S r_{i}$ work-to-home trip probability-distribution matrix.

The next equation locates the resulting incremental population in all of the subregions of the system:

$$
w^{(1)}(t)=\mathbf{L}_{t} e^{(1)}(t),
$$

where

$w^{(1)}(t)$ is an $R r \times 1$ vector of zonal populations due to the first iteration of the interregional model; and

$\mathbf{L}_{t} \quad$ is an $R r \times T S$ block-diagonal matrix whose typical diagonal block is equal to the matrix product $\mathbf{F}_{t}^{i} \mathbf{Q}^{i}$.

Substituting we find that

$$
e^{(1)}(t)=\mathrm{K}_{t} \mathrm{DAC}_{1} e^{(0)}(t)
$$

and

$$
w^{(1)}(t)=\mathbf{L}_{t} \mathbf{K}_{t} \mathbf{D A C}_{1} e^{(0)}(t) .
$$

At this stage the model reverts back to equation (14) for another iteration. We obtain the second round of production due to interregional demand from

$$
x^{(2)}(t)=\mathrm{AC}_{1} e^{(1)}(t) \text {, }
$$

which leads to

$$
e^{(2)}(t)=\left(\mathrm{K}_{t} \mathrm{DAC}_{1}\right)^{2} e^{(0)}(t)
$$

and

$$
w^{(2)}(t)=\mathbf{L}_{t}\left(\mathbf{K}_{t} \mathrm{DAC}_{1}\right)^{2} e^{(0)}(t) .
$$

More generally we have

$$
e^{(n)}(t)=\left(\mathrm{K}_{t} \mathrm{DAC}_{1}\right)^{n} e^{(0)}(t)
$$

and

$$
w^{(n)}(t)=\mathbf{L}_{t}\left(\mathbf{K}_{t} \mathrm{DAC}_{1}\right)^{n} e^{(0)}(t) .
$$

It follows that the total employment vector (all regions, all subregions, all sectors, and all iterations) is

$$
e(t)=\left[\mathrm{I}+\mathrm{K}_{t} \mathrm{DAC}_{1}+\ldots+\left(\mathrm{K}_{t} \mathrm{DAC}_{1}\right)^{n}+\ldots\right] e^{(0)}(t)=\left(\mathrm{I}-\mathrm{K}_{t} \mathrm{DAC}_{1}\right)^{-1} e^{(0)}(t) .
$$

After substitution from equation (13) this becomes

$$
e(t)=\left(\mathbf{I}-\mathbf{K}_{t} \mathbf{D A C}_{1}\right)^{-1} \mathbf{D} f(t),
$$


where $f(t)$ is obtained from equation (12). Then the population vector is

$$
w(t)=\mathbf{L}_{t}\left(\mathbf{I}-\mathbf{K}_{t} \mathrm{DAC}_{1}\right)^{-1} \mathbf{D} f(t) .
$$

Clearly relationships (27) and (28) appear as straightforward multiregional forms of relationships (4) and (5) of the classical Garin-Lowry model.

We now add the demographic side by use of the following components-of-change model (2) (Rogers, 1968):

$$
\boldsymbol{p}(t)=\mathrm{G}(t) \boldsymbol{p}(t-1),
$$

where $\mathbf{G}(t)$ is an $R \times R$ multiregional growth operator whose elements are functions of the exogenous regional birth and death rates as well as of the endogenous gross migration rates $m^{i j}(t)(j \neq i)$. These migration rates might be determined by variables reflecting differential opportunities between the origin and destination regions (for a summary of the relevant variables, see Greenwood, 1975). In view of the variables included in the model, we relate place-to-place migration rates to the difference (or ratio) of the origin and destination employment changes:

$$
m^{i j}(t)=m^{i j}\left[\frac{E^{i}(t-1)-E^{i}(t-2)}{E^{i}(t-2)}-\frac{E^{j}(t-1)-E^{j}(t-2)}{E^{j}(t-2)}\right],
$$

where $E^{i}(t-2)$ is the total regional employment of region $i$ at time $t-2$. This specification leaves the door open for the introduction of exogenous variables specific to the origin and destination.

Thus known variables determine total population at time $t$ through equations (29) and (30). By contrast, total employment is found by an iterative process similar to the one described in section 1 for just one metropolitan area.

The disaggregate vector of population can be consolidated by premultiplication by an $R \times T$ permutation matrix $\mathrm{C}_{2}$ into

$$
p(t)=\mathrm{C}_{2} \mathrm{~L}_{t}\left(\mathrm{I}-\mathrm{K}_{t} \mathrm{DAC}_{1}\right)^{-1} \mathrm{D} f(t) .
$$

If in each region zonal population-employment ratios keep the same structure over time, we have

$$
\mathrm{F}_{t}^{i}=\alpha_{t}^{i} \mathbf{F}_{t-1}^{i} .
$$

Substituting equation (32) into equation (31) and equating the resulting vector with the known value of $\boldsymbol{p}(t)$, we get a vector equation generalizing equation (11):

$$
\alpha_{t}=\left[\mathbf{C}_{2} \mathbf{L}_{t-1}\left(\mathbf{I}-\mathbf{K}_{t} \mathbf{D A C}_{1}\right)^{-1} \mathbf{D} f(t)\right]_{\mathrm{d}}^{-1} p(t),
$$

where $\alpha_{t}$ is an $R \times 1$ vector whose $i$ th element is $\alpha_{t}^{i}$ and where the notation $[y]_{\mathrm{d}}$ denotes a diagonal matrix whose diagonal is the vector $\boldsymbol{y}$.

To obtain $\alpha_{t}$, an iterative method can again be used. Calculate a first estimate $\alpha_{t}^{(1)}$ of $\alpha_{t}$ by replacing $\mathbf{K}_{t}$, which depends on $\alpha_{t}$, by $\mathbf{K}_{t-1}$ in the right-hand side of equation (33). For each $i$, plug the $i$ th element of $\alpha_{1}^{(1)}$ into equation (32) and the result into expression (17) to obtain a first estimate, $\mathbf{K}_{t}^{i(1)}$, of $\mathbf{K}_{t}^{i}$. Then calculate an improved estimate of $\alpha_{t}$ from equation (33), etc. This procedure is continued until convergence is achieved.

(2) In the case of an open country, equation (29) must be modified to account for international net in-migration (given exogenously):

$$
p(t)=\mathbf{G}(t) p(t-1)+I(t) .
$$




\section{Further demoeconomic considerations}

Up to this point our demoeconomic model allows for the derivation of employment (by sector) and for population estimates on three different levels: national (by aggregation of regional estimates), regional, and subregional (in the case of metropolitan areas). As suggested by Ledent (1978), the problem which inevitably emerges from the sole consideration of employment and population variables is the possibility of unreasonable values for the ratio of employment to population (and thus for the labor-force-participation rates) in each region. Yet, as already pointed out, a complete demoeconomic model includes the labor-force-participation rate, the unemployment rate, and the migration rate as endogenous and simultaneously determined variables. It is unlikely that these three are not determined within the same process of economic interaction and decisionmaking. Specifically we have five variables (employment, population, labor force, labor-force-participation rate, and unemployment rate), which are related by three equations and two identities (see figure 1).

Inevitably this means that two of the five variables have to be calculated as residuals. Perhaps the obvious candidates for residuals are the unemployment rate and the laborforce-participation rate since they are not primary variables. However, when they are calculated as residuals they often take on absurd values, especially the unemployment rate, and then the population and employment variables might not be consistent.

Thus, along with employment given by equation (27) and population given by equation (29), the unemployment rate is chosen as an endogenous variable (3). To explain it we could use an equation adapted from Glickman (1977) or Ledent (1978) such as

$$
u(t)=\gamma_{0}+\Gamma_{1} u(t-1)+\Gamma_{2} \Delta(t-1),
$$

where

$u(t-1)$ is an $R \times 1$ vector of unemployment rates, $\Delta(t-1)$ is an $R \times 1$ vector of relative employment increases, with general component

$$
[E(t-1)-E(t-2)] / E(t-1) \text {, and }
$$

$\gamma_{0}, \Gamma_{1}, \Gamma_{2}$ are a vector and two matrices of coefficients.

This means that total labor force and the labor-force-participation rate would be obtained as residuals.

We now have a complete dynamic and demoeconomic model for a system of regions where the subregional changes are endogenous, as are demographic changes. However, this model, which considers an adequate sectoral disaggregation on the economic side, is still aggregated on the demographic side. Its applicability is likely to be very limited unless it contains an age breakdown as suggested by the observation that the life cycle of a city and the life cycle of its population interact. Rapidly rising land values disrupt the lives of the elderly who rent housing. If they are numerous

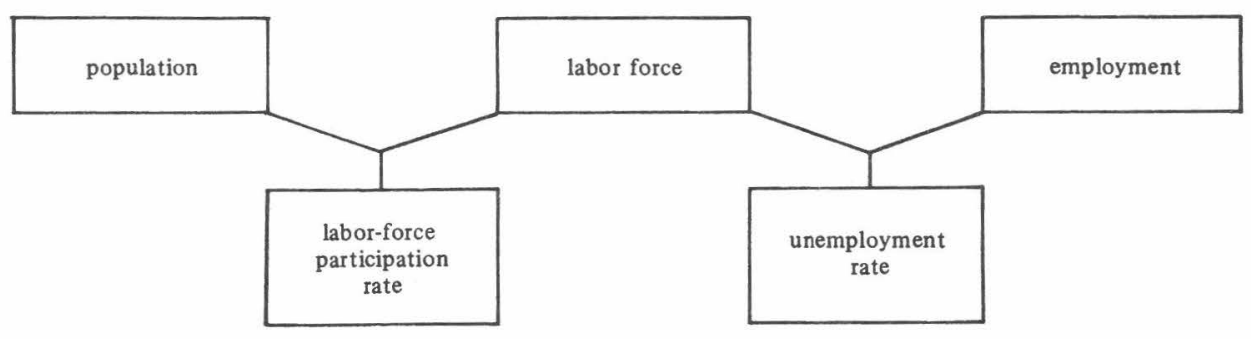

Figure 1. The basic relationship between the main demographic and economic variables in a consistent demoeconomic model (source: Ledent, 1978, page 547).

(3) Note that the consideration of unemployment rates in the model allows their inclusion as independent variables in the migration-rate equation (30). 
enough then rent controls may follow. As the population of a city ages, the school system becomes less important and the entire structure of social services changes. Transport modes emerge which feature escort- and demand-responsive features. Lawenforcement programs are affected where crime focuses on concentrations of the elderly. Likewise the association of revitalized city centers with a young urban population adds to the view that urban models that skip the age-specific dimension may not be useful.

How does the introduction of an age breakdown modify this model? First, concerning the economic side of the model, age structure can be easily introduced at the level of each regional Garin-Lowry model as shown by Schinnar (1978). Second, regarding the demographic side, the consideration of age-specific populations simply requires use of the disaggregate version of the components-of-change model (29), that is, the multiregional cohort-survival model (Rogers, 1975). Model (29) remains but $\mathrm{G}(t)$ is now an $R N \times R N$ multiregional growth operator whose elements depend on the age-specific (exogenous) fertility and mortality rates and (endogenous) mobility rates, where $N$ is the number of single-year age groups. Note that, if the regions are chosen such that distinctly urban and rural units are delineated, it is easy to make fertility and mortality rates endogenous (functions of the level of urbanization) and then to analyze the pace of the urbanization process.

Finally, to complete the disaggregate model, one needs to derive labor-forceparticipation rates by age groups. Since the total labor-force-participation rate is obtained as a residual, $N-1$ scalar equations concerning those age-specific rates are all that are required. Their expression is likely to be nonlinear for reasons set out in Ledent and Gordon (1978).

\section{Conclusions}

We have presented a model suggesting the evolution of metropolitan areas interacting through economic and demographic links, namely trade and migration respectively. The consideration of those links has moved us to follow the demoeconomic approach. This course allows the simultaneous determination of migration rates, labor-forceparticipation rates, and unemployment rates. Yet great care must be taken in the decision of which of these is to be exogenous. Further work should make commuting rates (considered exogenously in each metropolitan area) endogenous variables, because these are also a part of the decision framework which generates the three aforementioned rates.

Also our model includes an age breakdown of the population which indicates how urban structure can respond to a changing population structure. This gives foundation to a substantive discussion of systems of cities and their evolution. In brief, this model is suggestive of the demoeconomic approach advocated by Miron (1978) and Ledent and Gordon (1978), but also reveals the methodological difficulties that such an approach implies.

The empirical feasibility of implementing such a model, however, can be difficult. It is clear that its application to the urban system of most developed nations would lead to large data requirements in view of the large number of metropolitan areas existing in these nations. On the one hand one would need to have the parameters required by the implementation of each of the regional Garin-Lowry models. On the other hand one would desire the availability of an adequate interregional input-output table as well as time-series estimates of gross migration flows between each pair of metropolitan areas. Thus it is suggested that the framework first be implemented for a small country or a subdivision of a large nation, such as the state of California, being an open system and subdivided into four regions. 


\section{References}

Alperovich G, Bergsman J, Ehemann C, 1975 "An econometric model of employment growth in US metropolitan areas" Environment and Planning A 7 833-862

Alperovich G, Bergsman J, Ehemann C, 1977 "An econometric model of migration between US metropolitan areas" Urban Studies 14 135-145

Garin R A, 1966 "A matrix formulation of the Lowry model for intrametropolitan activity allocation" Journal of the American Institute of Planners 32 361-364

Glickman N, 1977 Econometric Analysis of Regional Analysis (Academic Press, New York)

Greenwood M J, 1975 "Research on internal migration in the United States: a survey" Journal of Economic Literature 12 397-433

Isard W, 1960 Methods of Regional Analysis (MIT Press, Cambridge, Mass)

Ledent J, 1978 "Regional multiplier analysis: a demometric approach" Environment and Planning $A$ $10537-560$

Ledent J, Gordon P, 1978 "A demoeconomic model of interregional growth rate differences" RM-78-52, International Institute for Applied Systems Analysis, Laxenburg, Austria, forthcoming in Geographical Analysis (January 1980)

Lowry I S, 1964 A Model of Metropolis RM-4035-RC, The Rand Corporation, Santa Monica, Calif.

Macgill S M, 1977 "The Lowry model as an input-output model and its extension to incorporate full intersectoral relations" Regional Studies 11 337-354

Miron J R, 1978 "Job-search perspectives on migration behaviour" Environment and Planning A 10 $519-535$

Rogers A, 1968 Matrix Analysis of Interregional Growth and Distribution (University of California Press, Berkeley, Calif.)

Rogers A, 1975 Introduction to Multiregional Mathematical Demography (John Wiley, New York)

Schinnar A P, 1978 "Invariant distributional regularities of nonbasic spatial activity allocations: the Garin-Lowry model revisited" Environment and Planning A 10 327-336 



\section{RELATED PUBLICATIONS ON URBAN CHANGE}

Niles Hansen, Human Settlement Systems: International Perspectives on Structure, Change and Public Policy. Ballinger, Cambridge, Massachusetts, 1978.

Andrei Rogers, Migration and Settlement: Selected Essays. RR-78-6. International Institute for Applied Systems Analysis, Laxenburg, Austria.

Andrei Rogers and Frans Willekens, Migration and Settlement: Measurement and Analysis. RR-78-13. International Institute for Applied Systems Analysis, Laxenburg, Austria.

Frans Willekens and Andrei Rogers, Spatial Population Analysis: Methods and Computer Programs. RR-78-18. International Institute for Applied Systems Analysis, Laxenburg, Austria.

L. Lacko, G. Enyedi, and G. Koszegfalvi, Functional Urban Regions in Hungary. CP-78-4. International Institute for Applied Systems Analysis, Laxenburg, Austria.

Peter Gordon, Deconcentration Without a "Clean Break". RM-78-39. International Institute for Applied Systems Analysis, Laxenburg, Austria. Also published in Environment and Planning A, 11(3):281-290.

Luis Castro and Andrei Rogers, Migration Age Patterns: I. Measurement and Analysis. WP-79-16. International Institute for Applied Systems Analysis, Laxenburg, Austria.

Lennart Ohlsson, Components of Urban Industrial Employment Change in a Small Open Economy: Sweden. WP-79-32. International Institute for Applied Systems Analysis, Laxenburg, Austria.

Lennart Ohlsson, Tracing Regional Patterns of Industrial Specialization Trends in Sweden. WP-79-33. International Institute for Applied Systems Analysis, Laxenburg, Austria.

Lennart Ohlsson, A Conceptual Framework for an Assessment of Swedish Regional Policy. WP-79-34. International Institute for Applied Systems Analysis, Laxenburg, Austria.

Andrei Rogers and Luis Castro, Migration Age Patterns: II. Cause-Specific Profiles. WP-79-65. International Institute for Applied Systems Analysis, Laxenburg, Austria. 
